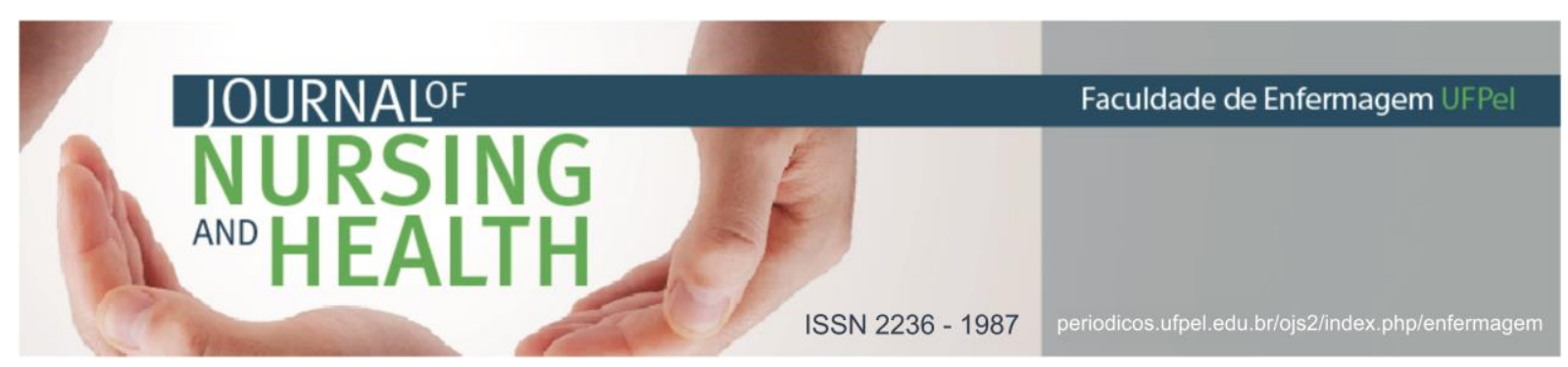

ARTIGO ORIGINAL

\title{
Percepções de gestantes tabagistas sobre malefícios do tabaco durante a gestação
}

\author{
Perceptions of pregnant women smokers on dangers of tobacco during pregnancy
Percepciones de las mujeres embarazadas fumadores sobre los peligros del tabaco durante el embarazo

Cruz, Joarez da ${ }^{1}$; Cruz Josceli Gonçalves da²; Bortoli, Cleunir de Fatima Candido de ${ }^{3}$

\begin{abstract}
RESUMO
Objetivo: conhecer a percepção das gestantes sobre os malefícios e as consequências do uso do tabaco durante a gestação. Método: estudo de campo, descritivo, qualitativo. Participaram do estudo 18 gestantes tabagistas. Os dados foram coletados, utilizando a técnica de entrevista semiestruturada. A análise dos dados foi orientada pela proposta operativa. Resultados: na análise, emergiram três categorias: início do tabagismo, que esteve relacionado ao entorno social e familiar; abordagem da gestante tabagista, revela a falta de apoio e suporte para o abandono do tabagismo; a percepção das gestantes sobre o tabagismo, que mesmo conhecendo os malefícios do tabaco, as dificuldades para abandonar o vício prevalecem. Considerações finais: o pré-natal é um importante momento para ações voltadas ao controle do tabagismo. São fundamentais a organização de grupos de apoio e o envolvimento do núcleo familiar, na cessação do tabagismo.
\end{abstract}

Descritores: Saúde da mulher; Gravidez; Hábito de fumar.

\begin{abstract}
Objective: the perception of pregnant women about the dangers and consequences of tobacco use during pregnancy. Method: field, descriptive, and qualitative study. Eighteen pregnant women smokers participated in this study. The data were collected using semi-structured interview technique. Data analysis was guided by the operational proposal. Results: from the analysis emerged three categories: beginning of smoking, which was related to the social and family environment; pregnant smoker's approach, reveals the lack of support for the abandonment of smoking; the perception of pregnant women about smoking, even knowing the harm of tobacco, the difficulties to abandon the habit prevails. Final considerations: prenatal care is an important moment for actions aimed at tobacco control. It is essential to the organization of support groups and the involvement of the nuclear family, in smoking cessation.
\end{abstract}

Descriptors: Women's health; Pregnancy; Smoking.

\footnotetext{
1 Enfermeiro. Especialista. Saúde e Segurança do Trabalho na Secretaria Municipal de Saúde, Pato Branco, Paraná, Brasil. E-mail: joarezdacruz@hotmail.com

2 Enfermeira. Enfermeira no Hospital Thereza Mussi. Pato Branco, Paraná, Brasil. E-mail: joenfermagem@hotmail.com

${ }^{3}$ Enfermeira. Mestre em Enfermagem. Enfermeira na Secretaria Municipal de Saúde de Pato Branco. Docente do curso de Enfermagem da Faculdade de Pato Branco - FADEP. Pato Branco, Paraná, Brasil. E-mail: cleunir_candido@hotmail.com
} 


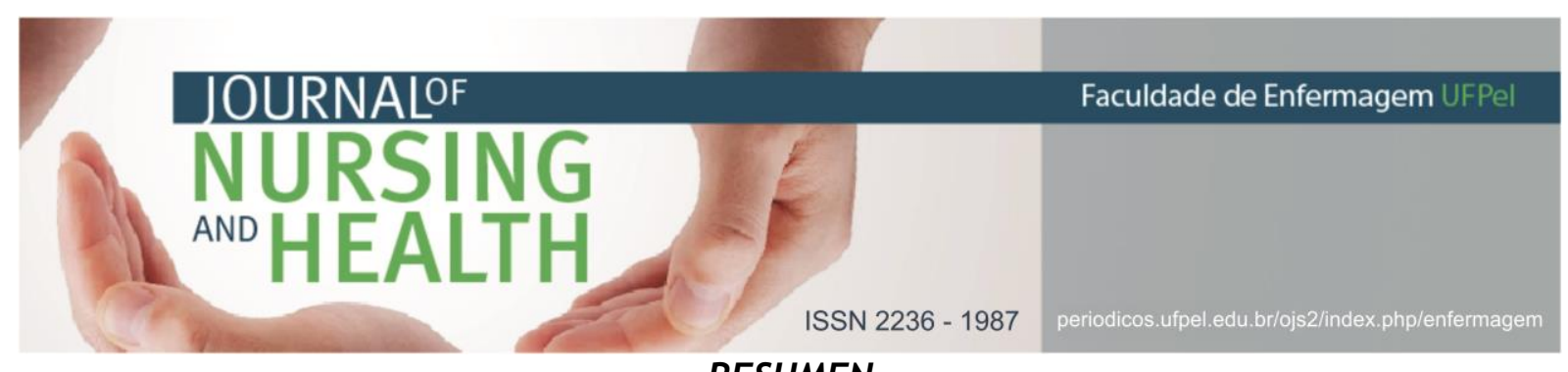

RESUMEN

Objetivo: conocer la percepción de las mujeres embarazadas acerca de los peligros y consecuencias del consumo de tabaco durante el embarazo. Método: estudio de campo descriptivo, cualitativo. Participaron en este estudio 18 mujeres embarazadas fumadoras. Los datos se recolectaron mediante la técnica de entrevista semiestructurada. Análisis de los datos fue guiado por la propuesta operativa. Resultados: el análisis, surgieron tres categorías: a partir del hábito de fumar, que se relacionó con el entorno social y familiar; enfoque de fumador embarazado, revela la falta de apoyo y ayuda para dejar de fumar; las dificultades para abandonar el hábito de la percepción de las mujeres embarazadas acerca de fumar, aún sabiendo el daña del tabaco, prevalece. Consideraciones finales: el cuidado prenatal es un momento importante para acciones encaminadas al control del tabaco. Es esencial para la organización de grupos de apoyo y la participación de la familia nuclear, para dejar de fumar.

Descriptores: Salud de la mujer; Embarazo; Hábito de fumar.

\section{INTRODUÇÃO}

O tabagismo é considerado uma das principais causas de doenças e agravos não transmissíveis, porém, preveníveis. $^{1}$ O uso do tabaco é responsável pelo desenvolvimento de diversos agravos de saúde, com aumento progressivo da sua prevalência no último século, resultando em altos custos sociais e econômicos. ${ }^{2}$

As estimativas de óbito por tabaco em 2030, será mais de 8 milhões de pessoas no mundo, e $80 \%$ dessas mortes prematuras ocorrerão em países em desenvolvimento. ${ }^{3}$ No Brasil, o tabagismo está relacionado a aproximadamente 200 mil mortes/ano, de acordo com as previsões, se tornará a principal causa de morte e invalidez, com mais de 10 milhões de óbitos por ano, fato este que o torna um problema social e de saúde pública. ${ }^{4}$ Os custos decorrentes do tabagismo, abrangem a assistência à saúde, aposentadorias precoces, pensões e benefícios pagos, poluição e degradação ambiental, incêndios e outros tipos de acidentes, pesquisa e educação, óbito de fumantes e não fumantes, além de sofrimento dos fumantes, não fumantes e seus familiares. ${ }^{5}$ Ainda, o país é responsável pela segunda causa de mortes entre as mulheres, com aproximadamente 26 mil mortes ao ano, totalizando $24 \%$ do total dos óbitos por cânceres, relacionados ao tabaco. ${ }^{6}$

Somado a isso, o tabagismo na gravidez atua negativamente em diversas fases da gestação, sendo que produtos derivados do cigarro, como o monóxido de carbono e a nicotina, passam facilmente pela placenta. Seu uso em período gestacional corresponde a fatores agravantes como: gravidez tubária, descolamento da placenta, má formação fetal, sangramento, aborto espontâneo, nascimento prematuro, neonatos com baixo peso, morte fetal ou mesmo do recém-nascido. ${ }^{7}$

O tabagismo materno representa uma das causas de nascimentos prematuros no Brasil. A prematuridade é o principal contribuinte para a mortalidade perinatal e infantil. É relevante o aumento da sobrevivência de pré-termos em países desenvolvidos, devido aos avanços tecnológicos e aos esforços de 


\section{JOURNALOF \\ NURSING \\ M०HEALTH}

\section{obstetras}

e

neonatologistas.

Entretanto, a redução da mortalidade é acompanhada de outro desafio, as sequelas relacionadas principalmente as funções cerebrais e pulmonares, comprometendo a qualidade de vida da criança. ${ }^{8}$

Diante do exposto, o presente estudo foi impulsionado pela seguinte questão de pesquisa: Qual a percepção das gestantes tabagistas, sobre as consequências do uso do tabaco durante a gestação? E como objetivo, conhecer a percepção das gestantes sobre os malefícios e as consequências do uso do tabaco durante a gestação.

\section{MATERIAIS E MÉTODOS}

A presente pesquisa caracterizouse por um estudo de campo, descritivo, de abordagem qualitativa. ${ }^{9}$ Desenvolvido em um município no sudoeste do Estado do Paraná, onde o cenário foi constituído por unidade básicas de saúde, assistidas por equipes Estratégia Saúde da Família (ESF).

Participaram do estudo, 18 gestantes, cujos critérios de inclusão elencados foram: ser tabagista e residir em áreas adscritas de ESF. Como critérios de exclusão: a idade menor de 18 anos.

Para a construção dos dados, utilizou-se da técnica de entrevista semiestruturada, sendo que as gestantes foram escolhidas de forma aleatoriamente e captadas através dos enfermeiros das equipes de ESF. 0 número total seguiu o critério de saturação dos dados, descrito como o conhecimento construído pelo pesquisador, no campo, que
ISSN 2236 - 1987

possibilitou a compreensão do grupo estudado. ${ }^{9}$

As entrevistas foram compostas por duas etapas: a primeira buscou-se caracterizar as participantes, com informações como idade, escolaridade e uso do tabaco durante as gestações. A segunda etapa foi constituída por questões orientadas pelo objetivo do estudo, sendo elas: Como iniciou com o cigarro? Você foi questionado se é fumante? Qual profissional? Foi ofertado algum tipo de ajuda para parar de fumar? Qual é a sua opinião sobre o tabagismo durante a gestação? Qual o conhecimento que possui sobre as consequências do tabagismo para o feto? A duração média de cada entrevista, foi de 15 minutos, sendo gravadas e posteriormente transcritas para análise. Aconteceram nas unidades de saúde e no domicílio das pesquisadas, com agendamento prévio.

Os dados foram analisados, orientados pela técnica de análise de conteúdo temática, pela proposta operativa, constituído em dois momentos operacionais. No primeiro, a fase exploratória, buscou-se a compreensão do contexto em que estavam inseridas as participantes. No segundo momento, denominado de interpretativo, ocorreu uma aproximação com os dados empíricos, compreendendo duas etapas: a ordenação, onde realizou-se a transcrição dos dados, a releitura e a organização do material; e a classificação, buscando-se o agrupamento dos dados, com a leitura horizontal e exaustiva dos registros, seguida da leitura transversal, a qual permitiu uma análise mais crítica do 
material, organizando-o por temas e a análise dos dados associada ao referencial teórico, elaborando assim, o relatório final. ${ }^{9}$

O estudo respeitou os princípios éticos, pautado pela Resolução $n^{\circ} 466 / 2012^{10}$, do Conselho Nacional de Saúde, relativa à pesquisa envolvendo seres humanos. As entrevistas foram realizadas após assinatura do Termo de Consentimento Livre e Esclarecido pelas participantes do estudo. Foi assegurado o sigilo e anonimato, sendo que as mesmas foram identificadas por um sistema alfanumérico, representado pela letra $G$, seguido de um numeral. A pesquisa obteve aprovação pelo Comitê de Ética em Pesquisa, em reunião realizada em 25 de setembro de 2013, com número de protocolo de pesquisa 185/13.

\section{RESULTADOS E DISCUSSÃO}

Participaram do estudo 18 gestantes tabagistas, com idade entre 18 e 43 anos, com maior concentração na faixa etária de 18 a 27 anos. Quanto ao grau de escolaridade, três não tinham concluído o ensino fundamental, uma possuía ensino fundamental completo, 13 delas possuíam ensino médio incompleto e apenas uma concluiu o ensino médio. Nenhuma possuía ensino superior.

Entre as pesquisadas, 11 relataram ter fumado em gestações anteriores, sendo que, o consumo menor que dez cigarros foi retratado por quatro gestantes, entre 11 e 20 cigarros foram quatro participantes, entre 21 e 30 cigarros apenas uma gestante e duas afirmaram fumar mais que 31 cigarros. Todas relataram uma frequência diária, no consumo do tabaco.

Entre as participantes, 12 delas afirmaram ter tentado parar de fumar, sendo que nenhuma obteve sucesso. Ainda, oito delas relatam ter problemas nas gestações anteriores, como: parto prematuro um caso, aborto cinco casos e recém-nascido com baixo peso dois casos.

$\mathrm{Na}$ residência das 18 gestantes tabagistas há 87 moradores, onde 32 são fumantes, e destes, 12 são os companheiros e 20 são os familiares. Quanto à distribuição da renda familiar, até um salário mínimo foram oito, entre um e dois salários mínimos, cinco, até três salários mínimos, duas, e acima de três salários, três participantes.

Analisando as narrativas das participantes, possibilitou elencar três categorias temáticas para discussão: início do tabagismo; a abordagem da gestante tabagista; e conhecimento das gestantes sobre o tabagismo.

\section{Início do tabagismo}

O tabagismo durante a gravidez é um evento que pode acarretar diversas complicações para o desenvolvimento gestacional. Para tanto, é fundamental a promoção de ações, visando a conscientização da população, sobretudo, a cessação do tabaco durante a gestação, não como atribuição restrita às campanhas de saúde pública, mas como uma causa de interesse e envolvimento de toda a sociedade. ${ }^{7}$

Analisando os relatos das gestantes pesquisadas neste estudo, observou-se que o início do uso do 
tabaco, ocorreu com companheiro ou com os próprios pais, sendo ainda alguns relatos de início no convívio social. Esses achados revelam a significância e a influência do meio social, como determinante para 0 início do tabagismo.

Foi com companheiro, eu estava muito mal acompanhada e aí comecei a beber, fumar tudo junto (G 01).

Bom, eu acendia o cigarro para a minha mãe. Eu comecei fumando aí, comecei o vício aí, fumando. Viciei e quando eu vi já era tarde, não consegui mais parar (G 02).

$\mathrm{Na}$ brincadeira no colégio, começamos com um, vamos fumar, vamos fumar que é bonito, dali foi indo (G 12).

\section{Pesquisando} gestantes brasileiras, autores identificaram uma associação positiva entre fumar e realizar menor número de consultas no pré-natal, ser fumante e conviver com companheiro fumante e fumar e possuir mais de um filho. Revelaram ainda, que o tabagismo possui maior tendência entre gestantes com menor escolaridade e aquelas que iniciaram o tabagismo precocemente. ${ }^{11}$

A influência familiar e do meio social, foram descritos como determinantes para o início do uso do tabaco entre jovens. Autores apontam que, jovens que possuem um familiar fumante, apresentam maior chance de iniciar o uso do tabaco. ${ }^{12} \mathrm{~A}$ influência do entorno social também é apontada como motivação para ingressar ao tabagismo, como forma de afirmação e pertença, perante ao grupo. ${ }^{13}$

Um estudo realizado com jovens, revelou que a idade entre 15 e 19 anos representa maiores chances de uso experimental de cigarros. A curiosidade, o efeito da substância (prazer e relaxamento), a influência dos amigos, constituíram as principais motivações identificadas para o início precoce do tabagismo. A mídia, a falta de esclarecimento dos pais para seus filhos e a correção dos pais de forma agressiva/coercitiva, também se mostraram determinantes para o início precoce do tabagismo. ${ }^{14}$

Os resultados do estudo revelam o meio social onde a gestante estava inserida, como determinante para sua iniciação ao tabagismo. Estes achados, corroboram com a literatura apresentada, reforçam o caráter social do hábito de fumar e o tabagismo enquanto problema de Saúde Pública.

\section{Abordagem da gestante tabagista}

Durante o período gestacional, a mulher ao realizar o acompanhamento pré-natal possui um contato mais frequente com a equipe de saúde, possibilitando o fortalecimento do vínculo entre usuárias e profissionais de saúde. As ações desenvolvidas na atenção pré-natal visam à promoção da saúde materna e infantil, buscando identificar os agravos à saúde e intervir de forma eficaz, reduzindo os danos à saúde do binômio mãe e filho. ${ }^{15}$

Quando identificado o tabagismo na gestação, a abordagem da gestante é um momento importante na redução e no controle do mesmo. Porém, tornase necessário o apoio e o suporte para a mesma durante este período, visando o sucesso na cessação do uso do tabaco. 


\section{JOURNALOF \\ NURSING \\ ANO HEALTH}

ISSN 2236 - 1987

diminuir os problemas relacionados aos efeitos do tabaco para o feto. ${ }^{11}$

No que se refere ao tratamento do tabagismo, autores reforçam o protagonismo dos sujeitos na promoção de sua saúde e o papel primordial dos serviços de saúde no atendimento aos usuários. Destacam que, as ações devem contemplar o acolhimento dos usuários de acordo com suas necessidades e transformando-os participantes ativos no controle do tabagismo. ${ }^{17}$

Neste contexto, o estudo reflete Não, ajuda assim eles não falaram. Só falaram que eu tinha que parar de fumar porque prejudica o bebê. A saúde da gente é tudo, só aconselharam, mais assim, em termos de ajuda não (G 05).

Fui orientada a parar pela Enfermeira, mas nunca fui atrás (G 08).

A gestação proporciona uma ampla oportunidade para a promover o abandono ao tabagismo. Esse ciclo da vida da mulher, deve ser contemplado como uma excelente oportunidade para fornecer mais orientações sobre os malefícios do tabagismo para a saúde, a fim de oferecer tratamento para cessação do tabagismo às usuárias. ${ }^{16}$

Durante o período gestacional, muitas mulheres tentam cessar o tabagismo, entre os principais motivos, estão a própria vontade de parar de fumar, a gravidez, a saúde do bebê e a saúde da gestante. 0 ponto de partida para parar de fumar é a crença em sua capacidade de resistir à vontade de parar de fumar, atitude que pode importância da organização do serviço no tratamento do tabagismo. Embora significativa, somente a abordagem da gestante não basta ao enfrentamento do tabagismo, pois mesmo diante do interesse da mesma em abandonar o hábito de fumar, fazse necessário dispor de recursos para auxiliá-la nesta etapa, promovendo sua autonomia e protagonismo no processo de cessação.

\section{Percepção das gestantes sobre o tabagismo}

Sabe-se que o tabagismo durante a gestação contribui para a prematuridade, baixo peso ao nascer, entre outros malefícios. Para tanto, é fundamental 0 planejamento da gravidez para que a futura mãe, caso utilize o tabaco, tenha acompanhamento para cessação do mesmo, deixando o vício gradativamente, antes de engravidar. Caso a gravidez não for planejada, também é indicado que ela pare de fumar gradativamente, uma vez que abstinência total do tabaco faz com que os componentes inalados presentes no organismo caiam na 


\section{JOURNALOF \\ NURSING \\ AND HEALTH

corrente sanguínea materna e causem danos ao bebê. ${ }^{18}$

Ao pesquisar a percepção das gestantes sobre os malefícios do tabaco, observa-se que as mesmas têm esclarecimentos sobre os efeitos do uso do cigarro durante a gestação. Analisando as narrativas, observa-se que o conhecimento já adquirido sobre os riscos não consegue superar a dificuldade em abandonar 0 tabagismo, como seguem as narrativas:

Eu, na minha opinião, faz muito mal para a criança. A primeira que eu tive (filha) é bem doente até hoje. Bronquite foi que a minha (filha) nasceu. Eu acho que foi pela causa do cigarro, da bebida também, que eu bebia bastante (G 01).

Complicado. A gente sabe que faz mal, a gente conhece os problemas que causa por causa do cigarro, mas a gente não tem força para se libertar. Porque a vontade de fumar vem e você sai desesperada (G 15).

Aborto, nascimento antes da hora, perca de peso do feto. Às vezes pode ter aborto bem antes, ou o feto pode nascer morto ou com alguma sequela (G 02).

Baixo peso e sei lá, acho que a criança pode nascer com problemas. Alguma deficiência, prematuro, antes do tempo pode prejudicar bastante. Não é que pode, prejudica sim e isso a gente sabe (G 09).
Pode nascer prematuro, pode ter problema de pulmão. Acho que é isso que eu sei ( $G$ 17).

Embora as gestantes reconheçam alguns malefícios, estes estão relacionados a uma maior preocupação com a saúde da criança, que compreendem principalmente doenças respiratórias, prematuridade e baixo peso ao nascer. No que se refere a saúde da mulher, observa-se uma lacuna no conhecimento das mesmas.

Em outras realidades, estudos apontam a carência de conhecimento das gestantes sobre os malefícios do tabaco na gestação. Onde, mesmo com alta prevalência de fumo ativo e passivo entre as gestantes, identificouse uma limitação do conhecimento das mesmas sobre as consequências de fumar para a saúde, quer seja durante a gestação ou em qualquer momento $\mathrm{da}$ vida da mulher. ${ }^{16} 0$ déficit de conhecimento das gestantes sobre as complicações, foi relacionado pelos autores, as escassas orientações de enfermagem durante acompanhamento pré-natal. ${ }^{19}$

Ainda que as participantes reconheçam os malefícios, identificase uma carência de medidas de apoio para cessação do tabagismo. Neste contexto, o aconselhamento por parte dos profissionais envolvidos possui papel essencial na promoção da saúde materna e infantil.

Autores reforçam que os profissionais da saúde devem buscar estratégias para intervenções efetivas durante o acompanhamento pré-natal. Entre essas ações, estão aquelas direcionadas e específicas para as gestantes, uma vez que essa população 


\section{JOURNALOF \\ NURSING \\ AND HEALTH}

ISSN 2236 - 1987

tabagismo, são insuficientes para o abandono do hábito de fumar. Mesmo conhecedoras das consequências, a dificuldade da cessação no período gestacional está presente em muitos relatos, o que pode ser atribuído também, às mudanças físicas e emocionais que ocorrem no organismo da mulher.

Propõem-se o desenvolvimento de grupos de apoio às gestantes tabagistas, organizado e coordenado pelas equipes de ESF. Para isso, as equipes possuem embasamento no Programa Nacional de Controle ao Tabagismo, o qual disponibiliza a metodologia de desenvolvimento dos grupos e material de apoio para esta ação.

Almeja-se, que os resultados deste estudo possam refletir na assistência prestada à gestante tabagista, durante o acompanhamento pré-natal, despertando entre os profissionais envolvidos, uma reflexão sobre sua prática diária. Ainda, que o uso do tabaco na gestação, seja reconhecido pelas equipes, como importante agravo a saúde materna e infantil.

Como limitações deste estudo, aponta-se o fato do número de participantes não possibilitar conhecer a prevalência do tabagismo entre essa população. Entretanto, propõem-se o desenvolvimento de novos estudos, buscando conhecer a prevalência do tabagismo em gestantes e seus determinantes nos desfechos das gestações, assim como a percepção dos profissionais sobre as práticas de cuidado às gestantes fumantes.

Somente o conhecimento e o esclarecimento sobre os malefícios do 


\section{REFERÊNCIAS}

1. Giron MPN, Souza DP, Fulco APL. Prevenção do tabagismo na adolescência: um desafio para a enfermagem. REME rev min enferm. 2010;14(4):587-94.

2. Ministério da Saúde (BR). Estratégias para o cuidado da pessoa com doença crônica: o cuidado da pessoa tabagista. Brasília; 2015.

3. Organização Mundial de Saúde. Relatório da OMS sobre a epidemia mundial do tabaco, 2011: alerta sobre os perigos do tabaco. Genebra; 2011.

4. Goulart D, Engroff P, Ely LS, Sgnaolin V, Santos EF, Terra NL, et al. Tabagismo em idosos. Rev bras geriatr gerontol. 2010;13(2):313-20.

5. Instituto Nacional do Câncer (BR). Organização Pan-Americana da Saúde. Pesquisa especial de tabagismo PETab: relatório Brasil. Rio de Janeiro: INCA; 2011.

6. Instituto Nacional de Câncer José Alencar Gomes da Silva. A situação do tabagismo no Brasil: dados dos inquéritos do Sistema Internacional de Vigilância, da Organização Mundial da Saúde, realizados no Brasil, entre 2002 e 2009. Rio de Janeiro: INCA; 2011.

7. Segura DCA, Missio L, Abentroth LRL, Pelenz M. Análise da frequência de partos prematuros relacionados ao uso do tabagismo durante a gravidez. Pleiade, Foz do Iguaçu. 2013;14(14):39-51.

8. Bettiol H, Barbieri MA, Silva AAM. Epidemiologia do nascimento prétermo: tendências atuais. Rev bras ginecol obstet [Internet]. 2010 [acesso em 2016 jun 25];32(2):57-60. Disponível em: http://www. producao.usp.br/bitstrea $\mathrm{m} /$ handle/BDPI/8080/art_BETTIOL_Ep idemiologia_do_nascimento_pretermo_tendencias_atuais_2010.pdf?se quence $=1$

9. Minayo MCS. O desafio do conhecimento: pesquisa qualitativa em saúde. $14^{\text {a }}$ ed. São Paulo: Hucitec; 2014.

10. Ministério da Saúde (BR). Resolução $\mathrm{n}^{\circ}$ 466, de 12 de dezembro de 2012 [Internet]. 2012 [acesso em 2013 set 30]. Disponível em: http://bvsms.saude.gov.br/bvs/saude legis/cns/2013/res0466_12_12_2012.h tml

11. Motta GCP, Echer IC, Lucena AF. Fatores associados ao tabagismo na gestação. Rev latino-am enfermagem. 2010;18(4):809-15.

12. Abreu MNS, Caiaffa WT. Influência do entorno familiar e do grupo social no tabagismo entre jovens brasileiros de 15 a 24 anos. Rev panam salud publica [Internet]. 2011 [acesso em 2016 jun 25];30(1):22-30. Disponível em:

http: / / www.scielosp.org/scielo.php?s cript=sci_arttext\&pid=S1020$49892011000700004 \& \operatorname{lng}=e n$

13. Oliveira CM, Gorayeb R. Diferenças de gênero e fatores motivacionais para início do tabagismo em adolescentes. Saude transform soc. 2012;3(1):49-54.

14. Machado Neto AS, Andrade TM, Napoli C, Abdon LCSL, Garcia MR, Bastos Fl. Determinantes da experimentação do cigarro e do início precoce do tabagismo entre adolescentes escolares em Salvador 


\section{JOURNALOF \\ NURSING \\ AND}

(BA). J bras pneumol. 2010;36(6):67482.

15. Ministério da Saúde (BR). Secretaria de Atenção à Saúde. Departamento de Atenção Básica. Atenção ao pré-natal de baixo risco. Brasília; 2012.

16. Bertani AL, Garcia T, Tanni SE, Godoy I. Prevenção do tabagismo na gravidez: importância do conhecimento materno sobre os malefícios para a saúde e opções de tratamento disponíveis. J bras pneumol [Internet]. 2015 [acesso em 2016 jun 25];41(2):175-81. Disponível em:

http://www.scielo.br/scielo.php?scrip $\mathrm{t}=\mathrm{sci}$ arttext\&pid=S1806-

$37132015000200175 \& \operatorname{lng}=e n$

17. Meier DAP, Vannuchi MTO, Secco IAO. Abandono do tratamento do tabagismo em programa de município do norte do paraná. Revista Espaço para a Saúde, Londrina. 2011;13(1):3544.

18. Egon SS, Schuertz KF, Paim MB, Zahra NM, Vaz RS. Gestantes: as consequências do uso do tabaco para o feto. Encontro de Bioética do Paraná Vulnerabilidades: pelo cuidado e defesa da vida em situações de maior vulnerabilidade. Curitiba; 2011.

19. Santos HTS, Oliveira GS, Soares PCF, Araújo WA, Almeida EUA, Oliveira MMLR. Os malefícios do uso do tabaco na gestação e suas complicações ao feto. Rev enferm UFPE on line [Internet]. 2015 [acesso em 2016 jun 25];9 Suppl:S9978-82. Disponível em: https://periodicos.ufpe.br/revistas/r evistaenfermagem/article/viewFile/1 0796/11958
ISSN 2236 - 1987

Data de submissão: 12/02/2016

Data de aceite: 25/01/2017

Data de publicação: 30/08/2017 